\title{
Large exchange bias in magnetic shape memory alloys by tuning magnetic ground state and magnetic-field history
}

\author{
Xiaoqi Liao ${ }^{1,2}$, Lumei Gao ${ }^{3}, \mathrm{Yu}_{\text {Wang }}{ }^{*}$, Xin $\mathrm{Xu}^{1}$, Muhammad Tahir Khan ${ }^{4}$, Tieyan Chang ${ }^{1}$, \\ Kaiyun Chen ${ }^{1}$, Yu-Jia Zeng ${ }^{2}$, Sen Yang ${ }^{1 *}$ and Peter Svedlindh ${ }^{5}$
}

\begin{abstract}
The exchange bias is of technological significance in magnetic recording and spintronic devices. Pursuing a large bias field is a long-term goal for the research field of magnetic shape memory alloys. In this work, a large bias field of $0.53 \mathrm{~T}$ is achieved in the $\mathrm{Ni}_{50} \mathrm{Mn}_{34} \mathrm{In}_{16-x} \mathrm{Fe}_{x}(x=1,3,5)$ system by tuning the magnetic ground state (determined by the composition $x$ ) and the magnetic-field history (determined by the magnetic field $H_{\mathrm{FC}}$ during field cooling and the maximum field $H_{\text {Max }}$ during isothermal magnetization). The maximum volume fraction of the interfaces between the ferromagnetic clusters and antiferromagnetic matrix and the strong interfacial interaction are achieved by tuning the magnetic ground state and the magnetic-field history, which results in strong magnetic unidirectional anisotropy and the large exchange bias. Moreover, two guidelines were proposed to obtain the large bias field. Firstly, the composition with a magnetic ground state consisting of the dilute spin glass and the strong antiferromagnetic matrix is preferred to obtain a large bias field; secondly, tuning the magnetic-field history by enhancing $H_{\mathrm{FC}}$ and reducing $H_{\mathrm{Max}}$ is beneficial to achieving large exchange bias. Our work provides an effective way for designing magnetically inhomogeneous compounds with large exchange bias.
\end{abstract}

Keywords: magnetic shape memory alloys, martensite, exchange bias, spin glass, antiferromagnetic

\section{INTRODUCTION}

The exchange bias (EB) phenomenon is characterized by a shift of the hysteresis loop along the magnetic field axis. Since its discovery in $\mathrm{Co} / \mathrm{CoO}$ fine particles by Meiklejohn and Bean in 1956 [1], various systems have been investigated to extend the material families with $\mathrm{EB}$, including multilayers [2], nanostructures [3], thin films [46], and bulk compounds with intrinsic phase separation [7]. The EB usually appears in an inhomogeneous magnetic state with ferromagnetic (FM) and antiferromagnetic (AFM) subsystems, which is attributed to the field-induced unidirectional magnetic anisotropy formed at the interfaces between the FM and AFM subsystems [8]. Pursuing a large bias field is a long-term goal of EB research because of its significant technological applications in ultrahigh-density magnetic recording, giant magnetoresistance, and spin-valve devices [9-11].

Off-stoichiometric Mn-rich Ni-Mn-Z ( $=$ = Sb, Sn, In, $\mathrm{Ga}$ ) magnetic shape memory alloys (MSMAs) have complicated magnetic states, which are suitable hosts for investigating EB. In these alloys, the FM interaction originates from the $\mathrm{Mn}-\mathrm{Mn}$ exchange interaction within the regular Mn sublattice and the AFM interaction originates from the Mn-Mn exchange interaction between the regular Mn sublattice and $\mathrm{Z}$ sublattice. The EB behaviours of these MSMAs vary greatly by changing compositions, because the FM and AFM interactions change considerably with the variation of compositions. As a consequence, different magnetic ground states, such as FM/ AFM [12], ferrimagnetic/AFM [13], and spin glass (SG)/ AFM states [14-16], may form, and the unidirectional

\footnotetext{
${ }^{1}$ MOE Key Laboratory for Nonequilibrium Synthesis and Modulation of Condensed Matter and State Key Laboratory for Mechanical Behavior of Materials, Xi'an Jiaotong University, Xi'an 710049, China

${ }^{2}$ College of Physics and Optoelectronic Engineering, Shenzhen University, Shenzhen 518060, China

${ }^{3}$ Instrument Analysis Center, Xi'an Jiaotong University, Xi'an 710049, China

${ }^{4}$ Faculty of Engineering and Applied Sciences, Department of Physics, RIPHAH International University I-14 Campus, Islamabad, Pakistan

${ }^{5}$ Solid State Physics, Department of Engineering Sciences, Ångström Laboratory, Uppsala University, 75121 Uppsala, Sweden

* Corresponding authors (emails: yuwang@mail.xjtu.edu.cn (Wang Y); Yang.Sen@mail.xjtu.edu.cn (Yang S))
} 
magnetic anisotropy at the interfaces between the FM clusters and the AFM matrix varies accordingly. Therefore, the magnetic ground states (corresponding to different compositions) of MSMAs play an important role in defining the EB properties.

The EB measurement follows a two-step protocol: i) cooling from a high temperature to the desired testing temperature under a constant magnetic field $\left(H_{\mathrm{FC}}\right)$, i.e., the field cooling (FC) process; ii) a subsequent isothermal magnetization process by sweeping a magnetic field to the maximum value $\left(H_{\mathrm{Max}}\right)$. Many investigations have demonstrated that the unidirectional anisotropy can be established below a blocking temperature during the FC process [17-31]. On the other hand, in some Ni-Mnbased MSMAs, the unidirectional anisotropy can also be isothermally created by applying a sufficiently large $H_{\text {Max }}$ after a zero-field cooling (ZFC) process [32-35]. A magnetic field-induced transition even occurs from a super SG to a superferromagnetic domain state during the isothermal magnetization process, which leads to spontaneous EB behaviour [34,35]. These experimental observations reveal that the magnetic-field histories (corresponding to $H_{\mathrm{FC}}$ and $H_{\mathrm{Max}}$ ) play a significant role on the EB behaviour.

As mentioned above, the EB is dependent on three key parameters: composition, $H_{\mathrm{FC}}$, and $H_{\mathrm{Max}}$. Numerous efforts have been devoted to achieve large EB in MSMAs by tuning these factors. In previous studies, only one or two of the three factors have been tuned to improve EB in separate MSMAs [27,34], which provides partial information on determining the bias field. The achievement of maximal EB requires tuning all of the above factors. However, a systematic study of the dependence of EB on these three factors in the same MSMA system is lacking so far. Thus, the guidelines for achieving large EB are far from being established.

To explore the effective means of obtaining large EB in MSMAs, the EB of $\mathrm{Ni}_{50} \mathrm{Mn}_{34} \mathrm{In}_{16-x} \mathrm{Fe}_{x}(x=1,3,5)$ was systematically studied with different magnetic ground states (corresponding to different $x$ ) and magnetic-field histories (corresponding to different $H_{\mathrm{FC}}$ and $H_{\mathrm{Max}}$ ). The $\mathrm{Ni}_{50} \mathrm{Mn}_{34} \mathrm{In}_{16}$ MSMA with a cluster SG (CSG)/AFM state [34] was chosen as the terminal alloy because it not only shows the EB effect, but also exhibits many other functional properties, such as giant magnetothermal conductivity [36], kinetic arrest [37], and the magnetocaloric effect [38]. The substitution of Fe for In can change the AFM interactions between the $\mathrm{Mn}$ and $\mathrm{In} / \mathrm{Fe}$ atoms so as to tune the magnetic ground state of the system. We found that the ground state of $\mathrm{Ni}_{50} \mathrm{Mn}_{34} \mathrm{In}_{16-x} \mathrm{Fe}_{x}$ changes from CSG/AFM to dilute SG (DSG)/AFM, as the Fe content $x$ changes from 1 to 5 . More importantly, the systematic measurements of EB behaviours with different $x, H_{\mathrm{FC}}$, and $H_{\mathrm{Max}}$ reveal that two guidelines should be orderly followed to obtain the large bias field. First, the composition with a magnetic ground state of DSG/AFM, in which small FM clusters embedded in a strong AFM matrix, is preferred to obtain large EB; second, tuning the magnetic-field history by enhancing $H_{\mathrm{FC}}$ and reducing $H_{\text {Max }}$ is beneficial to achieving large $\mathrm{EB}$, because the maximum volume fraction of FM/AFM interfaces and strong interface interaction can be obtained under these two conditions. This further results in strong unidirectional magnetic anisotropy and large EB. A maximum bias value of $0.53 \mathrm{~T}$ is obtained for $x=5$ under $H_{\mathrm{FC}}=$ $1.2 \mathrm{~T}$ and $H_{\mathrm{Max}}=1 \mathrm{~T}$. Our work contributes to the comprehensive understanding of the $\mathrm{EB}$ behaviour of bulk compounds with inhomogeneous magnetic phases, which provides an effective strategy for designing MSMAs with large EB.

\section{EXPERIMENTAL SECTION}

Polycrystalline ingots of nominal $\mathrm{Ni}_{50} \mathrm{Mn}_{34} \mathrm{In}_{16-x} \mathrm{Fe}_{x}(x=$ $1,3,5)$ MSMAs were prepared by arc melting stoichiometric amounts of the high-purity constituent elements under an Ar atmosphere. To achieve high homogeneity, the alloys were further annealed at $1173 \mathrm{~K}$ for $12 \mathrm{~h}$ in an evacuated quartz tube and then quenched in room-temperature water. Differential scanning calorimetry (DSC, TA instruments, Q2000) was used to determine the martensitic transition temperature with a temperature variation rate of $10 \mathrm{~K} \mathrm{~min}^{-1}$. Furthermore, $\mathrm{X}$-ray powder diffraction (XRD) was performed at room temperature to detect the structure of the samples by using a Bruker D8 Powder diffractometer with the $\mathrm{Cu} \mathrm{Ka} a_{1}$ line $\left(\lambda_{1}=1.5418\right.$ $\AA)$. The temperature dependence of the magnetization $(M-T)$, magnetic hysteresis $(M-H)$ loops, and alternating current (AC) susceptibility were measured by using a superconducting quantum interference device (Quantum Design, MPMS-XL-5) magnetometer. Two different protocols, namely, ZFC and FC, were adopted in the $M-T$ measurements. The ZFC magnetization was obtained by cooling a sample from 400 to $3 \mathrm{~K}$ in zero field and then applying a magnetic field of $10 \mathrm{mT}$ to measure the magnetization as the sample warmed up. The FC magnetization was subsequently obtained by measuring the magnetization with the same field of $10 \mathrm{mT}$ upon both cooling and heating. All of the $M-H$ loops were isothermally recorded with the measurement field $H_{\mathrm{Max}}$ at $5 \mathrm{~K}$ after cooling with a field $H_{\mathrm{FC}}$ from $400 \mathrm{~K}$. The tem- 
perature dependence of the AC susceptibility $\chi^{\prime}$ was measured using a sinusoidal magnetic field with an amplitude of $0.5 \mathrm{mT}$ and a frequency range from 1 to $333 \mathrm{~Hz}$

\section{RESULTS AND DISCUSSION}

The martensitic transformation of the $\mathrm{Ni}_{50} \mathrm{Mn}_{34} \mathrm{In}_{16-x} \mathrm{Fe}_{x}$ $(x=1,3,5)$ alloys was identified by DSC measurements, as shown in Fig. 1a-c. The DSC curves of these samples display large exothermic and endothermic peaks with distinct thermal hysteresis upon cooling and heating procedures, revealing the occurrence of the martensitic transformation. The martensitic transformation temperature $T_{\mathrm{M}}$ is indicated by the arrows (Fig. $1 \mathrm{a}-\mathrm{c}$ ). It shifts to higher temperatures with increasing $\mathrm{Fe}$ content $x$. Fig. 1d-f present the ZFC and FC curves of the samples, which characterize their magnetic transition behaviours. The ZFC and FC curves of all the samples show an abrupt increase at high temperatures, demonstrating the existence of the FM phase in austenite. Its FM transition temperature is denoted by $T_{\mathrm{c}}$ (Fig. $1 \mathrm{~d}-\mathrm{f}$ ). After slight cooling below $T_{c}$, the ZFC and FC curves exhibit a sharp drop at $T_{\mathrm{M}}$ due to the martensitic transition. In the lowtemperature martensitic region, the ZFC curves of the samples exhibit a peak at $T_{\mathrm{p}}$, below which the ZFC and FC curves deviate from each other. This demonstrates the existence of a magnetic freezing transition. It has been shown that AFM interactions are greatly enhanced below the martensitic transition in Mn-rich Ni-Mn-In MSMAs [39], leading to a sharp drop of magnetization at $T_{\mathrm{M}}$. Moreover, weak FM interactions may also exist in the martensitic state [40], which give rise to the formation of many FM clusters coexisting with the surrounding AFM matrix. Owing to the spin frustration caused by the inherent competition between FM and AFM interactions, these FM clusters undergo a freezing transition into the SG state below $T_{\mathrm{p}}[19,27,30]$.

The freezing transition of the $\mathrm{Ni}_{50} \mathrm{Mn}_{34} \mathrm{In}_{16-x} \mathrm{Fe}_{x}(x=1$, $3,5)$ samples was further characterized by the temperature dependence of AC magnetic susceptibility $\chi^{\prime}$
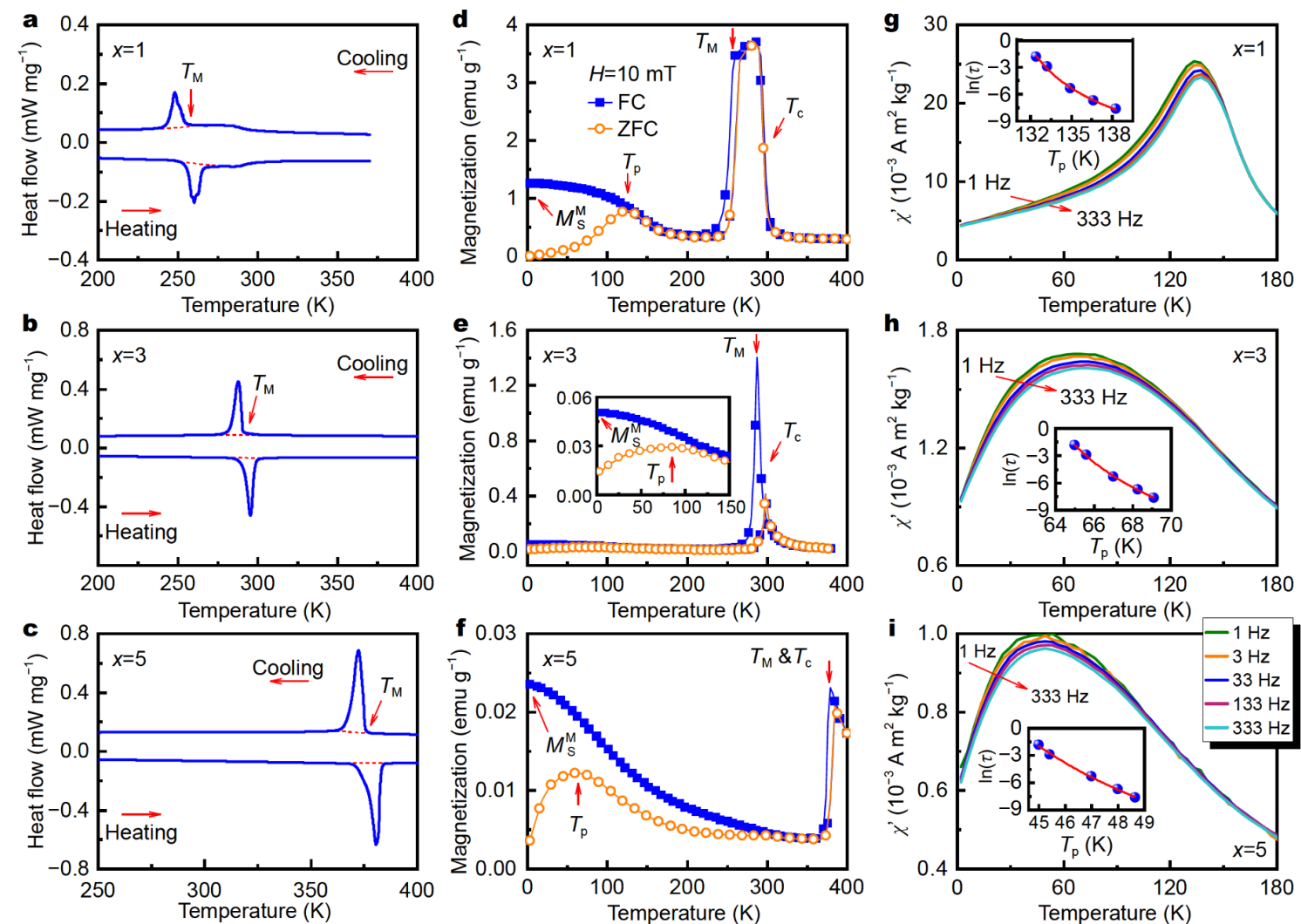

Figure 1 DSC curves of $\mathrm{Ni}_{50} \mathrm{Mn}_{34} \mathrm{In}_{16-x} \mathrm{Fe}_{x}$ MSMA for (a) $x=1$, (b) $x=3$, and (c) $x=5$. Temperature dependence of magnetization curves with ZFC (open circles) and FC (solid squares) protocols under a field of $10 \mathrm{mT}$ for (d) $x=1$, (e) $x=3$, and (f) $x=5$. Real part ( $\chi$ ) of AC susceptibility $v s$. temperature for (g) $x=1$, (h) $x=3$, and (i) $x=5$, which was measured in a frequency range of $1-333 \mathrm{~Hz}$ at a magnetic field strength of 0.5 mT. Insets of $(\mathrm{g}-\mathrm{i})$ show that the $\ln \tau$ vs. $T_{\mathrm{p}}$ (solid spheres) curve conforms to the power-law (red line). 
(Fig. 1g-i). The susceptibility peak temperature $T_{\mathrm{p}}$ displays apparent frequency dispersion for all the three samples, which is the signal of the SG transition. The frequency dependence of $T_{\mathrm{p}}$ for the SG transition conforms to the critical-slowing-down power law $\tau=$ $\tau_{0}\left(T_{\mathrm{p}} / T_{\mathrm{g}}-1\right)^{-z v}[27,30]$. In this formula, $\tau$ is the relaxation time of locally correlated spins and equals $(2 \pi f)^{-1}$ ( $f$ is the frequency); $\tau_{0}, T_{\mathrm{g}}$, and $z v$ are the microscopic relaxation time, the SG transition temperature, and the dynamic critical exponent, respectively. As shown in the insets in Fig. $1 g-i$, the dependence of $\ln \tau$ on $T_{p}$ for the three samples can be well fitted by the critical-slowing-down power law. The $\tau_{0}$ values of these samples obtained by fitting are plotted in Fig. $2 \mathrm{a}$ and decreases from $\sim 10^{-9}$ to $\sim 10^{-11} \mathrm{~s}$ as $x$ increases from 1 to 5 . It was reported that $\tau_{0}$ is approximately $10^{-9} \mathrm{~s}$ for CSG $[34,35]$, while it lies in the range of $\sim 10^{-12}$ to $10^{-14} \mathrm{~s}$ for DSG [41-43]. Thus, the results in Fig. 2a reveal the low-temperature glassy state of $\mathrm{Ni}_{50} \mathrm{Mn}_{34} \mathrm{In}_{16-x} \mathrm{Fe}_{x}$ changes from CSG to DSG with increasing $x$.

The variation of the low temperature glassy state is due to the change of AFM interaction of the $\mathrm{Ni}_{50} \mathrm{Mn}_{34} \mathrm{In}_{16-x} \mathrm{Fe}_{x}$ system, which is achieved by tuning the lattice parameter through the substitution of Fe for In. The room-temperature XRD patterns (Fig. S1, SI) show the L2 1 parent phase for $x=1$ and the six-layered modulated (6M) monoclinic martensite phase for $x=5$ [44]. The L2 lat- tice parameter ( $a=5.999 \AA$ ) obtained from the Rietveld refinement for $\mathrm{Ni}_{50} \mathrm{Mn}_{34} \mathrm{In}_{15} \mathrm{Fe}_{1}(x=1)$ is smaller than that reported for $\mathrm{Ni}_{50} \mathrm{Mn}_{34} \mathrm{In}_{16}(a=6.006 \AA)$ [36]. Such a decrease in the lattice parameter is due to the substitution of small-radius Fe for large-radius In atoms. With the increase of $x$, the lattice parameter of the system gradually decreases, which is beneficial to promoting the AFM interaction between the regular Mn sublattice and the In/Fe sublattice in its martensite phase. This characteristic is also consistent with the results in Fig. $2 \mathrm{~b}$, where the saturated magnetization of martensite $\left(M_{\mathrm{S}}{ }^{\mathrm{M}}\right)$ at $5 \mathrm{~K}$ considerably decreases with increasing $x$. Since the AFM interaction becomes stronger, the volume fraction of the AFM matrix extends and the size of the FM clusters shrinks correspondingly, resulting in the gradual change from CSG into DSG with increasing $x$.

The phase diagram in Fig. $2 c$ summarises the evolution of the structural transformations and the magnetic phases as a function of $x$ for the $\mathrm{Ni}_{50} \mathrm{Mn}_{34} \mathrm{In}_{16-x} \mathrm{Fe}_{x}$ system. The substitution of Fe for In shifts $T_{\mathrm{M}}$ to a higher temperature, which agrees with previous results for $\mathrm{Ni}-\mathrm{Mn}-\mathrm{In}-\mathrm{Fe}$ MSMAs [45]. $T_{\mathrm{c}}$ of austenite insignificantly changes within $1 \leq x<3$, while it overlaps with $T_{\mathrm{M}}$ for $3<x \leq 5$. The low temperature magnetic state for $x=1$ is CSG/ AFM with large FM clusters embedded in the AFM matrix (Fig. 2d). The clusters of the CSG become smaller (Fig. 2e) for $x=3$. The low temperature magnetic state for
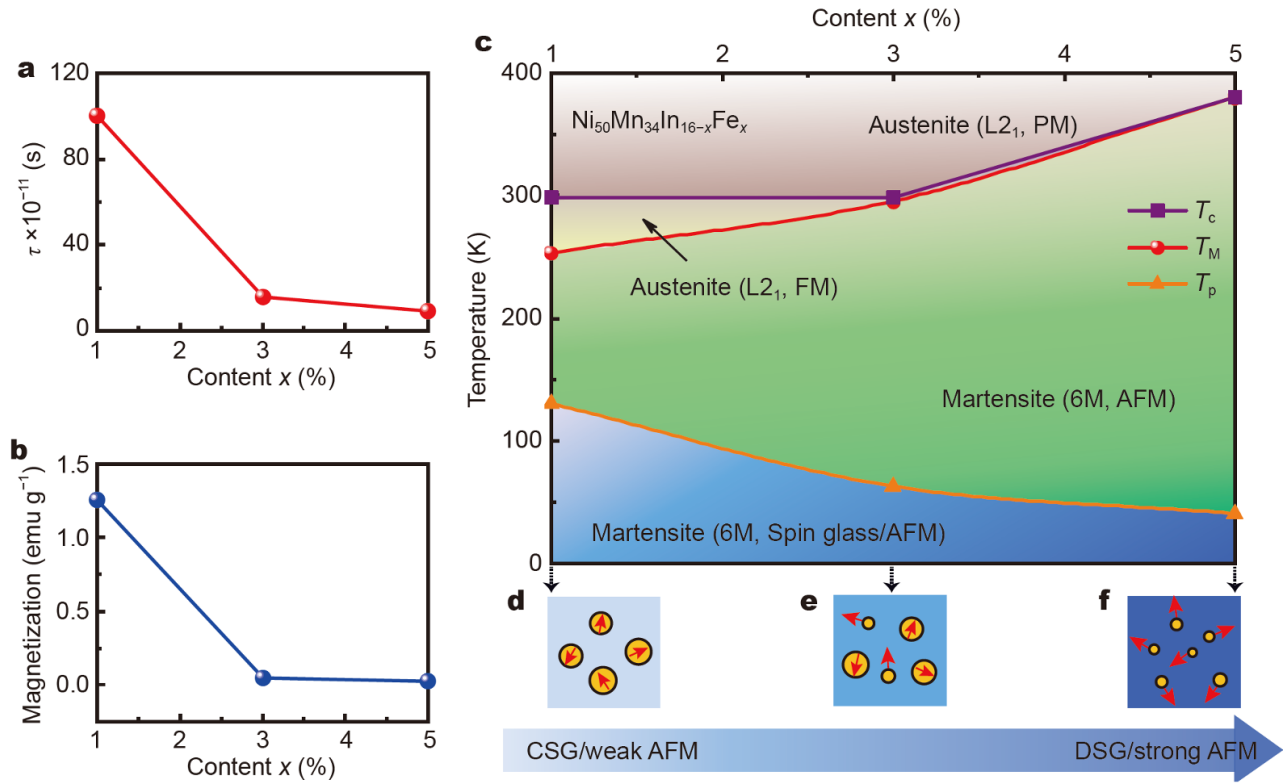

Figure 2 (a) Dependence of $\tau_{0}$ on $x$ obtained by fitting the AC susceptibility in Fig. 1. (b) Saturated magnetization $\left(M_{\mathrm{S}}^{\mathrm{M}}\right)$ in martensite at $5 \mathrm{~K}$ as a function of $x$. (c) Phase diagram for $\mathrm{Ni}_{50} \mathrm{Mn}_{34} \mathrm{In}_{16-x} \mathrm{Fe}_{x}(x=1-5)$ MSMAs. (d-f) show schematically that the low-temperature magnetic state evolves from CSG/AFM to DSG/AFM as $x$ increases from 1 to 5 . The yellow circles represent the FM clusters with different sizes. The blue background represents the AFM matrix, the color of which gradually becomes darker, representing the AFM interaction becoming stronger. 
$x=5$ changes to DSG/AFM, where many atomic spins are embedded in the AFM matrix (Fig. 2f). The SG transition temperature $T_{\mathrm{g}}$ rapidly decreases with increasing $x$, which is consistent with the fact that large clusters tend to freeze at higher temperatures.

Fig. 3 displays the dependence of the $M-H$ curves on the Fe content $x$ (corresponding to the magnetic ground states), $H_{\mathrm{FC}}$, and $H_{\mathrm{Max}}$ (corresponding to the magneticfield history) for the $\mathrm{Ni}_{50} \mathrm{Mn}_{34} \mathrm{In}_{16-x} \mathrm{Fe}_{x}$ system. All $M-H$ curves were measured at $5 \mathrm{~K}$. The $M-H$ curves for different $x$ under the same $H_{\mathrm{FC}}(0.4 \mathrm{~T})$ and $H_{\mathrm{Max}}(1 \mathrm{~T})$ are compared in Fig. 3a. The inset reveals that all the three samples exhibit EB with the $M-H$ curves shifted toward the negative magnetic field axis. The increase in $x$ changes the $M-H$ curves from an FM-like shape to a paramagnetic (PM)-like shape, because the change of magnetic state from CSG/AFM to DSG/AFM is accompanied with a considerable weakening of magnetism. Fig. $3 \mathrm{~b}$ compares the $M-H$ curves under different $H_{\mathrm{FC}}$ but with the same $x$ (3) and $H_{\text {Max }}(1 \mathrm{~T})$. Its $M-H$ curve changes from PM-like to FM-like shape and its EB behaviour also changes greatly with increasing $H_{\mathrm{FC}}$, because the $\mathrm{FM}$ subsystems are strengthened and the corresponding magnetization is enhanced by increasing $H_{\mathrm{FC}}$. The $M-H$ curves for different $H_{\mathrm{Max}}$ but with the same $x$ (3) and $H_{\mathrm{FC}}(0.4 \mathrm{~T})$ are compared in Fig. 3c. Interestingly, the shapes of the $M-H$ curves are similar but their shifts are quite different for various $H_{\mathrm{Max}}$. This characteristic demonstrates that $H_{\mathrm{Max}}$ can also change the magnetic state and thus affects the EB behaviour of the system.

In order to obtain a better understanding of the dependence of $\mathrm{EB}$ on $x, H_{\mathrm{FC}}$, and $H_{\mathrm{Max}}$, the bias field $H_{\mathrm{EB}}$ as a function of $H_{\mathrm{FC}}$ for different $H_{\mathrm{Max}}$ and $x$ are depicted in Fig. $4 \mathrm{a}$. $H_{\mathrm{EB}}$ was calculated by using $H_{\mathrm{EB}}=-\left(H_{L}+H_{R}\right) / 2$, where $H_{\mathrm{L}}$ and $H_{\mathrm{R}}$ are the left and right coercive fields, respectively. $H_{\mathrm{EB}}$ increases up to a maximum bias value $\left(H_{\mathrm{MEB}}\right)$ and then decreases with increasing $H_{\mathrm{FC}}$, forming a peak shape of the $H_{\mathrm{EB}}$ vs. $H_{\mathrm{FC}}$ curves. The $H_{\mathrm{EB}}$ increases with increasing $H_{\mathrm{Max}}$ when $H_{\mathrm{FC}}$ is very small, but it is obviously reduced by $H_{\mathrm{Max}}$ when $H_{\mathrm{FC}}$ is relatively large. Moreover, the shape of the $H_{\mathrm{EB}}$ vs. $H_{\mathrm{FC}}$ curve changes from a sharp peak to a broadened profile.

The dependence of EB on $x, H_{\mathrm{FC}}$, and $H_{\mathrm{Max}}$ (Fig. $4 \mathrm{a}$ ) can be understood as the follows. After the FC process, the random moments of FM clusters or spins become aligned along the positive $H_{\mathrm{FC}}$ direction. The unidirectional anisotropy can be created at the interface between FM clusters and the AFM matrix, because the AFM matrix exerts a pinning effect on the FM clusters at the interface through the interaction between them $[1,11]$. Evidently, the unidirectional anisotropy at the interface and its associated $H_{\mathrm{EB}}$ is determined by two factors: i) the volume fraction of the FM/AFM interface, which relies on the average size of the FM clusters and the distance between FM clusters; ii) the strength of the FM/AFM interface interaction.

The change of $H_{\mathrm{EB}}$ with $H_{\mathrm{FC}}$ (dot dash line of Fig. $4 \mathrm{~b}$ ) can be explained by Fig. $4 \mathrm{~b}(1-3)$, which show the influence of $H_{\mathrm{FC}}$ on the magnetic state under the small $H_{\mathrm{Max}}$. With increasing $H_{\mathrm{FC}}$, the average size of the FM clusters grows, thus the volume fraction of the FM/AFM interface increases, leading to the enhancement of $H_{\mathrm{EB}}$. However, $H_{\mathrm{EB}}$ cannot monotonously increase with $H_{\mathrm{FC}}$, since the increase in $H_{\mathrm{FC}}$ not only causes cluster growth, but also reduces the distance between clusters and even makes them merge with each other, which reduces the volume fraction of FM/AFM interfaces and the corresponding $H_{\mathrm{EB}}$. In the low- $H_{\mathrm{FC}}$ region, the average size of the FM clusters is small and the distance between them is large (Fig. $4 \mathrm{~b}(1)$ ), the growth of the volume fraction of the FM/
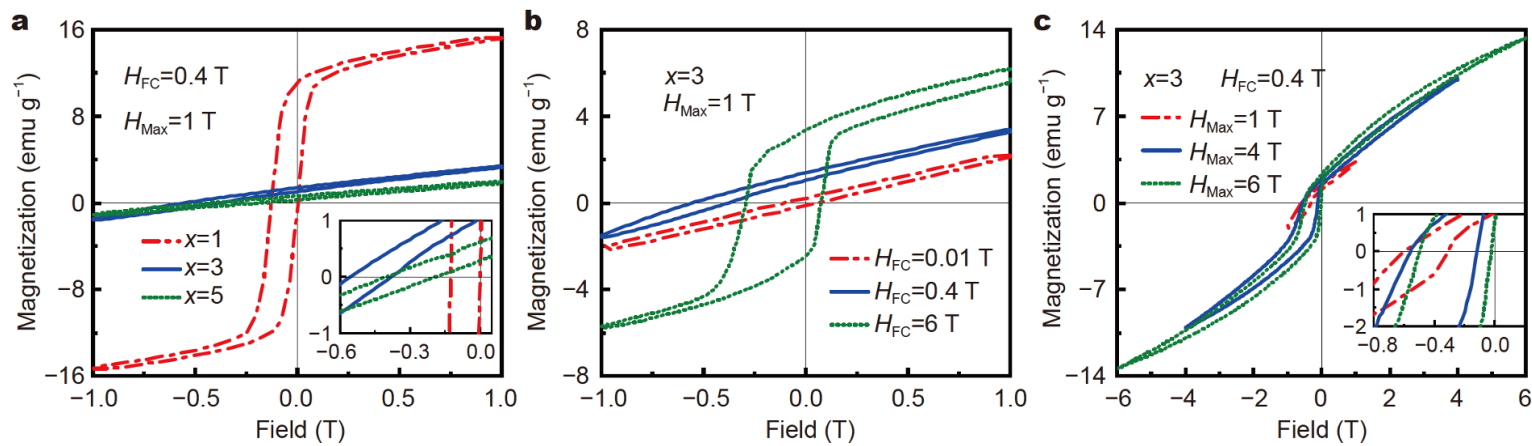

Figure $3 M-H$ curves of $\mathrm{Ni}_{50} \mathrm{Mn}_{34} \mathrm{In}_{16-x} \mathrm{Fe}_{x}(x=1,3,5)$ MSMAs measured at $5 \mathrm{~K}$. (a) $M-H$ curves measured with $H_{\mathrm{FC}}=0.4 \mathrm{~T}$ and $H_{\mathrm{Max}}=1 \mathrm{~T}$ for $x=$ 1, 3, 5. (b) $M-H$ curves measured with $x=3$ and $H_{\mathrm{Max}}=1 \mathrm{~T}$ for $H_{\mathrm{FC}}=0.01,0.4$, and $6 \mathrm{~T}$. (c) $M-H$ curves obtained with $x=3$ and $H_{\mathrm{FC}}=0.4 \mathrm{~T}$ for $H_{\text {Max }}=1,4$, and $6 \mathrm{~T}$. A magnified view of the low-field region is depicted in the inset of (a) and (c). 

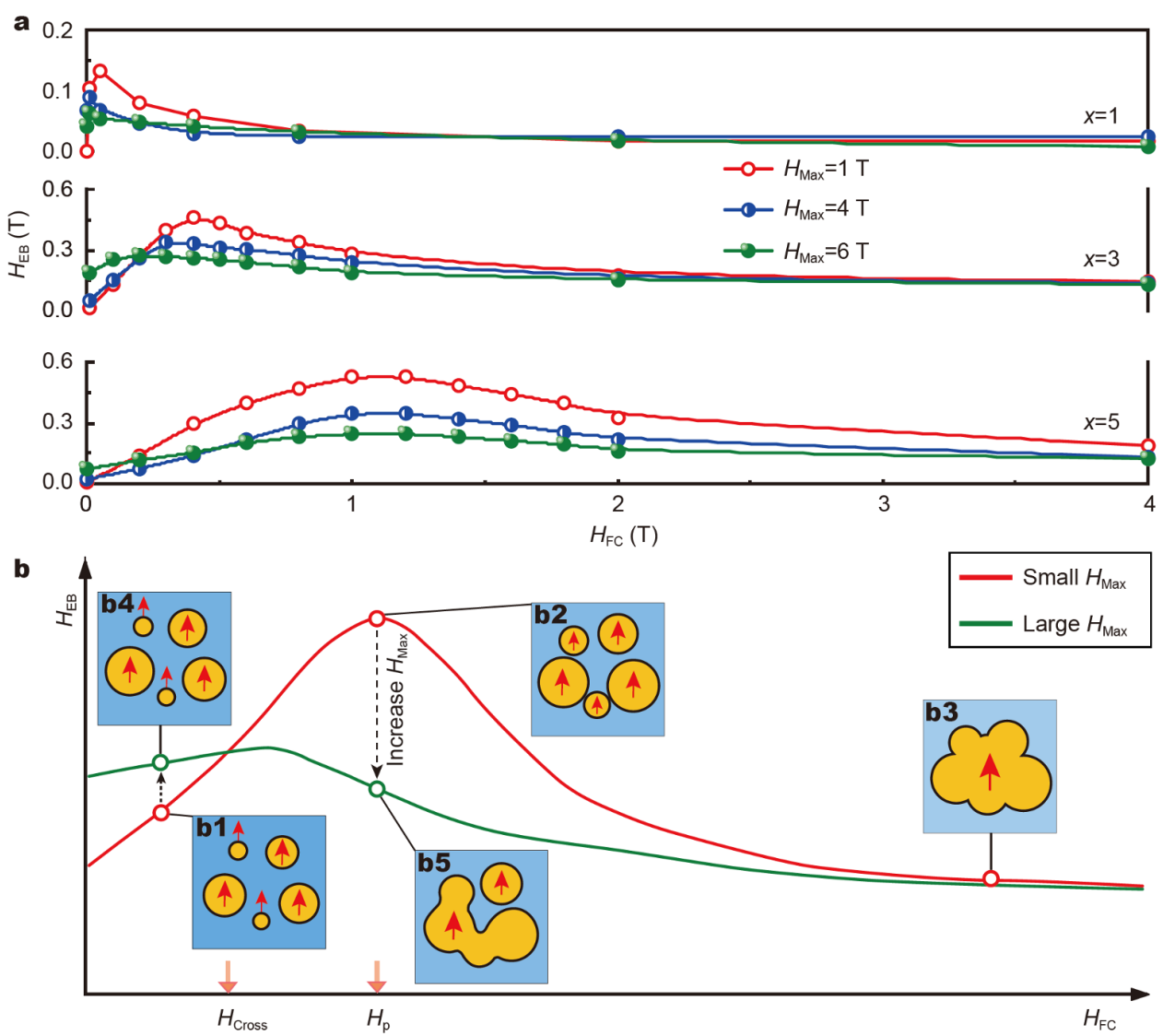

Figure 4 (a) $H_{\mathrm{EB}}$ as a function of $H_{\mathrm{FC}}$ for $\mathrm{Ni}_{50} \mathrm{Mn}_{34} \mathrm{In}_{16-x} \mathrm{Fe}_{x}(x=1,3,5)$ MSMAs measured under $H_{\mathrm{Max}}=1,4$, and $6 \mathrm{~T}$. (b) Sketches for the evolution of magnetic state from (1) to (5) with varying $H_{\mathrm{FC}}$ and $H_{\mathrm{Max}}$, which explains the dependence of $\mathrm{EB}$ on $H_{\mathrm{FC}}$ and $H_{\mathrm{Max}}$. The yellow circles represent the FM clusters with different sizes and the blue background represents the AFM matrix.

AFM interface plays a dominant role in determining $H_{\mathrm{EB}}$. However, the average cluster size becomes quite large and a considerable number of clusters merge (Fig. $4 \mathrm{~b}(2$ and 3)), when $H_{\mathrm{FC}}$ exceeds a critical value $\left(H_{\mathrm{p}}\right)$. For such a case, the decrease of volume fraction of the FM/AFM interface plays a dominant role in determining $H_{\mathrm{EB}}$. Therefore, $H_{\mathrm{EB}}$ vs. $H_{\mathrm{FC}}$ shows an increase below the $H_{\mathrm{p}}$ but a decrease above it.

The changes of $H_{\mathrm{EB}}$ with $H_{\mathrm{Max}}$ can be interpreted by Fig. $4 \mathrm{~b}\left(4\right.$ and 5), which show the effects of $H_{\mathrm{Max}}$ on the magnetic state. In the very small $H_{\mathrm{FC}}$ region $\left(<H_{\text {Cross }}\right)$, the FM clusters are small and the distance between them is large (Fig. $4 \mathrm{~b}(1)$ ). Most of them grow but do not touch with each other when $H_{\text {Max }}$ is applied (Fig. $4 \mathrm{~b}(4)$ ). Thus, the volume fraction of the FM/AFM interface and corresponding $H_{\mathrm{EB}}$ become larger. However, in the larger $H_{\mathrm{FC}}$ region ( $>H_{\text {Cross }}$ ), a considerable number of $\mathrm{FM}$ clusters grow up and become close. The corresponding magnetic state under large $H_{\mathrm{FC}}$ and small $H_{\mathrm{Max}}$ is schematically displayed in Fig. 4b(2). The FM clusters merge by applying large $H_{\text {Max }}$ (Fig. $\left.4 \mathrm{~b}(5)\right)$, which reduces the volume fraction of the FM/AFM interface and the corresponding $H_{\mathrm{EB}}$.

The dependence of $H_{\mathrm{MEB}}$ on $x$ can be explained by Fig. $2 \mathrm{~d}-\mathrm{f}$, which displays the change of magnetic state with $x$. For a certain composition $x$, its largest bias field $H_{\mathrm{MEB}}$ corresponds to the optimized volume fraction of the FM/AFM interface obtained by tuning $H_{\mathrm{FC}}$ and $H_{\mathrm{Max}}$. However, the strength of the FM/AFM interaction at the interface is quite different for different compositions. With increasing $x$, the AFM interaction in the matrix becomes much stronger, which exerts a stronger pinning effect on FM clusters. This promotes strong interfacial unidirectional anisotropy, resulting in a large $H_{\mathrm{MEB}}$.

Based on the data in Fig. $4 \mathrm{a}, H_{\mathrm{MEB}}$ of $\mathrm{Ni}_{50} \mathrm{Mn}_{34} \mathrm{In}_{16-x} \mathrm{Fe}_{x}$ $(x=1,3,5)$ is plotted as functions of $x, H_{\mathrm{FC}}$, and $H_{\mathrm{Max}}$ in Fig. 5 . These results clearly reveal that the optimized value of $H_{\mathrm{MEB}}$ is jointly determined by the three parameters ( $x$, $H_{\mathrm{FC}}$, and $\left.H_{\mathrm{Max}}\right)$. For a fixed composition $x$, the combination of an increase in $H_{\mathrm{FC}}$ and a decrease in $H_{\mathrm{Max}}$ is 


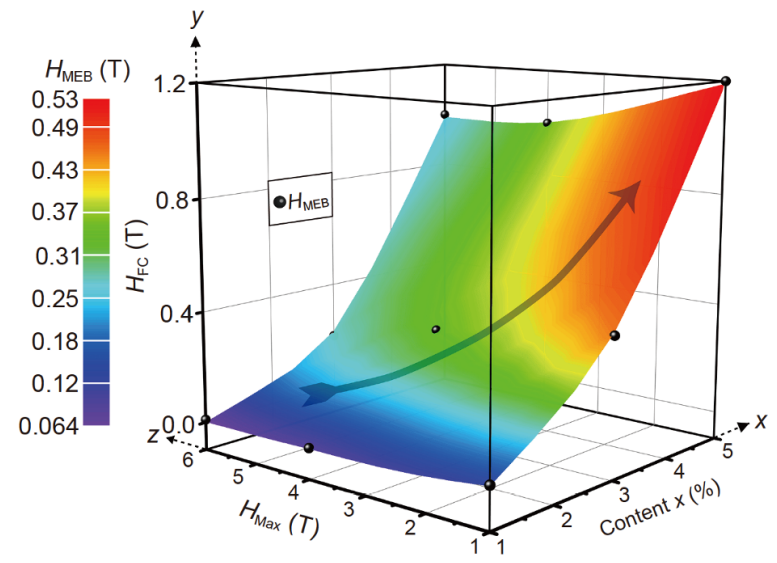

Figure 5 Three-dimensional contour for $H_{\mathrm{MEB}}$ (black spheres) as the function of $x, H_{\mathrm{FC}}$, and $H_{\mathrm{Max}}$.

beneficial to achieving large EB, because the maximum FM/AFM interface can be obtained in such a magneticfield history. Furthermore, $H_{\mathrm{MEB}}$ shifts to higher values, when $x$ changes from 1 to 5 . The $x=5$ sample with DSG/ AFM state shows large $H_{\mathrm{MEB}}$, and its $H_{\mathrm{MEB}}$ increases rapidly by tuning $H_{\mathrm{FC}}$ and $H_{\mathrm{Max}}$. By contrast, the $x=1$ sample with CSG/AFM shows much smaller $H_{\mathrm{MEB}}$, and its $H_{\text {MEB }}$ increases slowly for the same situation. This demonstrates that a DSG/AFM state with small FM clusters embedded in a strong AFM matrix is more capable of achieving large EB. The guidance for obtaining large EB is visually indicated by an arrow on the contour surface of Fig. 5, which can be described as the following two points: i) the composition with a magnetic ground state of DSG/ AFM is preferred to obtain large EB; ii) tuning the magnetic-field history by enhancing $H_{\mathrm{FC}}$ and reducing $H_{\mathrm{Max}}$ is beneficial to achieving large EB. The maximum bias field of $0.53 \mathrm{~T}$ is obtained for $x=5$ under $H_{\mathrm{FC}}=1.2 \mathrm{~T}$ and $H_{\mathrm{Max}}=1 \mathrm{~T}$. The maximum $H_{\mathrm{EB}}$ of the $x=5$ sample $\left(\mathrm{Ni}_{2}\right.$ $\left.\mathrm{Mn}_{1.36} \mathrm{In}_{0.44} \mathrm{Fe}_{0.2}\right)$ with respect to its optimized $H_{\mathrm{FC}}$ and $H_{\text {Max }}$ is compared with those of some important MSMAs in Fig. 6. As indicated by a red pentagram, its maximum $H_{\mathrm{EB}}$ is much larger than those of other MSMAs.

\section{CONCLUSIONS}

In this study, the EB behaviours of $\mathrm{Ni}_{50} \mathrm{Mn}_{34} \mathrm{In}_{16-x} \mathrm{Fe}_{x}(x=$ $1,3,5)$ MSMAs have been systematically investigated by tuning the magnetic ground states (composition $x$ ) and magnetic-field histories $\left(H_{\mathrm{FC}}\right.$ and $\left.H_{\mathrm{Max}}\right)$. The following conclusions were obtained: (1) the ground state changes from the CSG/AFM to DSG/AFM as $x$ increases from 1 to 5 , because the substitution of $\mathrm{Fe}$ for In decreases the lattice parameter of the system, which leads to the en-

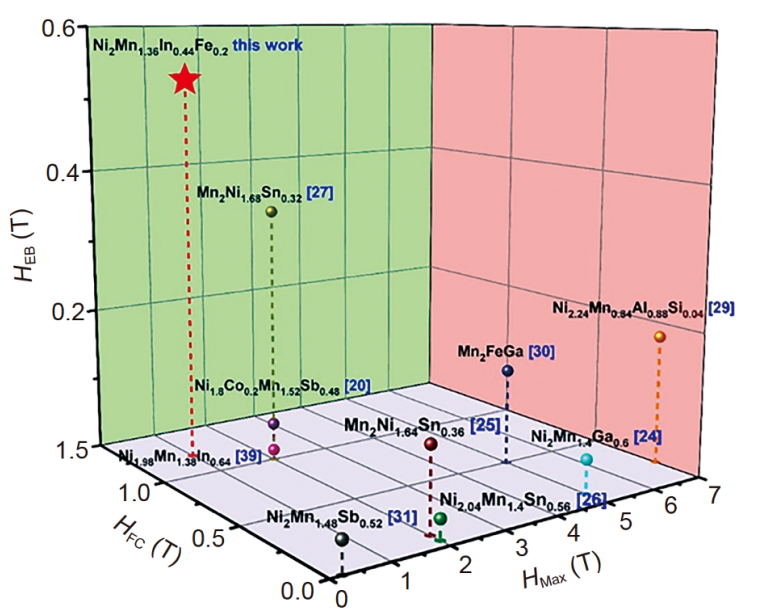

Figure 6 Plot of $H_{\mathrm{EB}}$ with respect to $H_{\mathrm{FC}}$ and $H_{\mathrm{Max}}$ for important MSMAs.

hancement of the AFM interaction and the size reduction of FM clusters; (2) through tuning the magnetic ground states (composition $x$ ) and magnetic-field histories $\left(H_{\mathrm{FC}}\right.$ and $H_{\mathrm{Max}}$ ), the volume fraction of FM/AFM interfaces and the strength of interface interaction can be optimized, which leads to strong unidirectional magnetic anisotropy at FM/AFM interfaces and large EB. A large bias field of $0.53 \mathrm{~T}$ is achieved for $x=5, H_{\mathrm{FC}}=1.2 \mathrm{~T}$, and $H_{\mathrm{Max}}=1 \mathrm{~T}$; (3) two guidelines should be utilized to realize large EB: i) the composition with a magnetic ground state of DSG/ AFM consisting of small FM clusters and strong AFM matrix is preferred; ii) tuning magnetic-field history by enhancing $H_{\mathrm{FC}}$ and reducing $H_{\mathrm{Max}}$. These guidelines provide an effective way for producing large EB in the systems with inhomogeneous magnetic phases.

Received 11 February 2020; accepted 24 February 2020; published online 8 April 2020

1 Meiklejohn WH, Bean CP. New magnetic anisotropy. Phys Rev, 1956, 102: 1413-1414

2 Nogués J, Sort J, Langlais V, et al. Exchange bias in nanostructures. Phys Rep, 2005, 422: 65-117

3 Phan MH, Alonso J, Khurshid H, et al. Exchange bias effects in iron oxide-based nanoparticle systems. Nanomaterials, 2016, 6: 221

4 Berkowitz AE, Takano K. Exchange anisotropy - a review. J Magn Magn Mater, 1999, 200: 552-570

5 Li H, Wang C, Li D, et al. Magnetic orders and origin of exchange bias in Co clusters embedded oxide nanocomposite films. J PhysCondens Matter, 2019, 31: 155301

6 Liu HL, Brems S, Zeng YJ, et al. Interplay between magnetocrystalline anisotropy and exchange bias in epitaxial $\mathrm{CoO} / \mathrm{Co}$ films. J Phys-Condens Matter, 2016, 28: 196002

7 Giri S, Patra M, Majumdar S. Exchange bias effect in alloys and compounds. J Phys-Condens Matter, 2011, 23: 073201

8 Takano K, Kodama RH, Berkowitz AE, et al. Interfacial uncompensated antiferromagnetic spins: role in unidirectional ani- 
sotropy in polycrystalline $\mathrm{Ni}_{81} \mathrm{Fe}_{19} / \mathrm{CoO}$ Bilayers. Phys Rev Lett, 1997, 79: 1130-1133

9 Kools JCS. Exchange-biased spin-valves for magnetic storage. IEEE Trans Magn, 1996, 32: 3165-3184

10 Gider, Runge, Marley, et al. The magnetic stability of spindependent tunneling devices. Science, 1998, 281: 797-799

11 Nogués J, Schuller IK. Exchange bias. J Magn Magn Mater, 1999, 192: 203-232

12 Wang RL, Lee MK, Xu LS, et al. Effect of thermal cycle on the interfacial antiferromagnetic spin configuration and exchange bias in Ni-Mn-Sb alloy. AIP Adv, 2012, 2: 032181

13 Nayak AK, Nicklas M, Chadov S, et al. Design of compensated ferrimagnetic Heusler alloys for giant tunable exchange bias. Nat Mater, 2015, 14: 679-684

14 Nayak AK, Nicklas M, Chadov S, et al. Large zero-field cooled exchange-bias in bulk $\mathrm{Mn}_{2} \mathrm{PtGa}$. Phys Rev Lett, 2013, 110: 127204

15 Ray MK, Maji B, Modak M, et al. Magnetic ground state and giant spontaneous exchange bias in $\mathrm{Ni}_{46} \mathrm{Mn}_{43} \mathrm{In}_{11}$ alloy. J Magn Magn Mater, 2017, 429: 110-116

16 Jia L, Shen J, Li M, et al. Tuning antiferromagnetic exchange interaction for spontaneous exchange bias in MnNiSnSi system. APL Mater, 2017, 5: 126105

17 Leighton C, Nogués J, Jönsson-Åkerman BJ, et al. Coercivity enhancement in exchange biased systems driven by interfacial magnetic frustration. Phys Rev Lett, 2000, 84: 3466-3469

18 Morales R, Li ZP, Olamit J, et al. Role of the antiferromagnetic bulk spin structure on exchange bias. Phys Rev Lett, 2009, 102: 097201

19 Chatterjee S, Giri S, De SK, et al. Reentrant-spin-glass state in $\mathrm{Ni}_{2} \mathrm{Mn}_{1.36} \mathrm{Sn}_{0.64}$ shape-memory alloy. Phys Rev B, 2009, 79: 092410

20 Nayak AK, Suresh KG, Nigam AK. Observation of enhanced exchange bias behaviour in NiCoMnSb Heusler alloys. J Phys D-Appl Phys, 2009, 42: 115004

21 Nayak AK, Sahoo R, Suresh KG, et al. Anisotropy induced large exchange bias behavior in ball milled Ni-Co-Mn-Sb alloys. Appl Phys Lett, 2011, 98: 232502

22 Ma L, Wang WH, Lu JB, et al. Coexistence of reentrant-spin-glass and ferromagnetic martensitic phases in the $\mathrm{Mn}_{2} \mathrm{Ni}_{1.6} \mathrm{Sn}_{0.4}$ Heusler alloy. Appl Phys Lett, 2011, 99: 182507

23 Yang YB, Ma XB, Chen XG, et al. Structure and exchange bias of $\mathrm{Ni}_{50} \mathrm{Mn}_{37} \mathrm{Sn}_{13}$ ribbons. J Appl Phys, 2012, 111: 07A916

24 Han ZD, Qian B, Wang DH, et al. Magnetic phase separation and exchange bias in off-stoichiometric Ni-Mn-Ga alloys. Appl Phys Lett, 2013, 103: 172403

25 Sharma J, Suresh KG. Observation of large exchange bias effect in bulk $\mathrm{Mn}_{50} \mathrm{Ni}_{41} \mathrm{Sn}_{9}$ Heusler alloy. IEEE Trans Magn, 2014, 50: 1-4

26 Pramanick S, Chatterjee S, Giri S, et al. Excess Ni-doping induced enhanced room temperature magneto-functionality in Ni-Mn-Sn based shape memory alloy. Appl Phys Lett, 2014, 105: 112407

27 Sharma J, Suresh KG. Observation of giant exchange bias in bulk $\mathrm{Mn}_{50} \mathrm{Ni}_{42} \mathrm{Sn}_{8}$ Heusler alloy. Appl Phys Lett, 2015, 106: 072405

28 Sharma J, Suresh KG. Investigation of multifunctional properties of $\mathrm{Mn}_{50} \mathrm{Ni}_{40-x} \mathrm{Co}_{x} \mathrm{Sn}_{10}(x=0-6)$ Heusler alloys. J Alloys Compd, 2015, 620: 329-336

29 Singh R, Ingale B, Varga LK, et al. Large exchange bias in polycrystalline ribbons of $\mathrm{Ni}_{56} \mathrm{Mn}_{21} \mathrm{Al}_{22} \mathrm{Si}_{1}$. J Magn Magn Mater, 2015, 394: $143-147$

30 Liu ZH, Zhang YJ, Zhang HG, et al. Giant exchange bias in $\mathrm{Mn}_{2} \mathrm{FeGa}$ with hexagonal structure. Appl Phys Lett, 2016, 109: 032408

31 Yang YB, Liu SQ, Zhao $\mathrm{H}$, et al. Magnetic structure and phase transition of $\mathrm{Ni}_{2} \mathrm{Mn}_{1.48} \mathrm{Sb}_{0.52}$ magnetic shape memory compound. Scripta Mater, 2016, 116: 31-35

32 Wang X, Li MM, Li J, et al. Design of anti-site disorder for tunable spontaneous exchange bias: $\mathrm{Mn}-\mathrm{Ni}-\mathrm{Al}$ alloys as a case. Appl Phys Lett, 2018, 113: 212402

33 Dubiel $€$, Żywczak A, Maziarz W, et al. Magnetic phase transition and exchange bias in $\mathrm{Ni}_{45} \mathrm{Co}_{5} \mathrm{Mn}_{35.5} \mathrm{In}_{14.5}$ Heusler alloy. Appl Magn Reson, 2019, 50: 809-818

34 Wang BM, Liu Y, Ren P, et al. Large exchange bias after zero-field cooling from an unmagnetized state. Phys Rev Lett, 2011, 106: 077203

35 Liao $\mathrm{P}$, Jing $\mathrm{C}$, Wang $\mathrm{XL}$, et al. Strongly enhanced antiferromagnetism and giant spontaneous exchange bias in $\mathrm{Ni}_{50} \mathrm{Mn}_{36} \mathrm{Co}_{4} \mathrm{Sn}_{10}$ Heusler alloy. Appl Phys Lett, 2014, 104: 092410

36 Zhang B, Zhang XX, Yu SY, et al. Giant magnetothermal conductivity in the Ni-Mn-In ferromagnetic shape memory alloys. Appl Phys Lett, 2007, 91: 012510

37 Sharma VK, Chattopadhyay MK, Roy SB. Kinetic arrest of the first order austenite to martensite phase transition in $\mathrm{Ni}_{50} \mathrm{Mn}_{34} \mathrm{In}_{16}$ : $\mathrm{dc}$ magnetization studies. Phys Rev B, 2007, 76: 140401

38 Chattopadhyay MK, Sharma VK, Roy SB. Thermomagnetic history dependence of magnetocaloric effect in $\mathrm{Ni}_{50} \mathrm{Mn}_{34} \mathrm{In}_{16}$. Appl Phys Lett, 2008, 92: 022503

39 Wang BM, Liu Y, Wang L, et al. Exchange bias and its training effect in the martensitic state of bulk polycrystalline $\mathrm{Ni}_{49.5} \mathrm{Mn}_{34.5} \mathrm{In}_{16}$. J Appl Phys, 2008, 104: 043916

40 Enkovaara J, Heczko O, Ayuela A, et al. Coexistence of ferromagnetic and antiferromagnetic order in $\mathrm{Mn}$-doped $\mathrm{Ni}_{2} \mathrm{MnGa}$. Phys Rev B, 2003, 67: 212405

41 Malinowski A, Bezusyy VL, Minikayev R, et al. Spin-glass behavior in Ni-doped $\mathrm{La}_{1.85} \mathrm{Sr}_{0.15} \mathrm{CuO}$. Phys Rev B, 2011, 84: 024409

42 Tian F, Cao K, Zhang Y, et al. Giant spontaneous exchange bias triggered by crossover of superspin glass in $\mathrm{Sb}$-doped $\mathrm{Ni}_{50} \mathrm{Mn}_{38} \mathrm{Ga}_{12}$ Heusler alloys. Sci Rep, 2016, 6: 30801

43 Zhao DW, Li GK, Wang SQ, et al. Tuning exchange bias by Co doping in $\mathrm{Mn}_{50} \mathrm{Ni}_{41-\chi} \mathrm{Sn}_{9} \mathrm{Co}_{x}$ melt-spun ribbons. J Appl Phys, 2014, 116: 103910

44 Yan H, Zhang Y, Xu N, et al. Crystal structure determination of incommensurate modulated martensite in Ni-Mn-In Heusler alloys. Acta Mater, 2015, 88: 375-388

45 Lobo DN, Priolkar KR, Emura S, et al. Ferromagnetic interactions and martensitic transformation in Fe doped Ni-Mn-In shape memory alloys. J Appl Phys, 2014, 116: 183903

Acknowledgements This work was supported by the National Natural Science Foundation of China (51471127, 51431007 and 51371134), the Program for Young Scientific New-star in Shaanxi Province of China (2014KJXX-35), the Innovation Capability Support Program of Shaanxi (2018PT-28 and 2017KTPT-04), Shenzhen Science and Technology Project (JCYJ20180507182246321), and the Fundamental Research Funds for Central Universities of China.

Author contributions Liao $\mathrm{X}$ and Wang $\mathrm{Y}$ designed the experiments; Gao L, Xu X, Chang T and Chen K performed the experiments; Liao X, Wang Y, Zeng Y and Svedlindh P performed the data analysis; Liao X, Wang Y, Khan MT and Yang S wrote and revised the paper. All authors contributed to the general discussion.

Conflict of interest The authors declare that they have no conflict of interest. 
Supplementary information Supporting data are available in the online version of the paper.

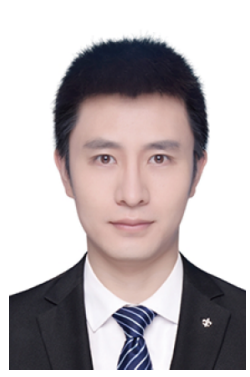

Xiaoqi Liao is a postdoctoral researcher at Shenzhen University. He received a $\mathrm{PhD}$ degree in 2019 from Xi'an Jiaotong University (XJTU). During 2017-2018, he was a joint-training PhD student at Uppsala University. His research interest focuses on magnetic materials and devices, including magnetic shape memory alloys, selfassembly of magnetic nanoparticles and 2D materials.

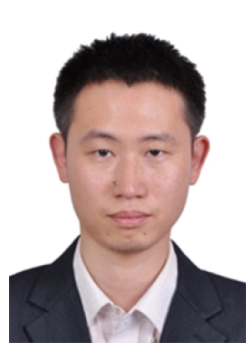

Yu Wang received his $\mathrm{PhD}$ degree (2008) from XJTU in the fields of condensed matter physics. Afterwards, he spent two years at the National Institute for Materials Science of Japan as a postdoctor under the Japan Society for the Promotion of Science (JSPS) Fellowship. In 2019, he was appointed as a full professor at the School of Science, XJTU. His research interests include magnetic materials, shape memory alloys and spintronics.

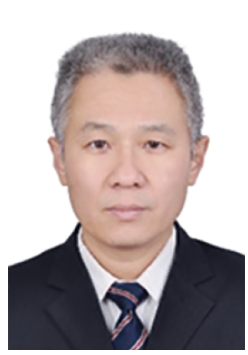

Sen Yang received his $\mathrm{PhD}$ degree in materials physics from XJTU, China in 2005. He joined the National Institute for Materials Science, Japan in 2005 as a JSPS post-doctor. In the year of 2010, he came back to XJTU and was promoted to full professor in 2013. His research interests are in magnetism and magnetic materials, smart materials, phase transition and so on.

\section{基于磁性形状记忆合金磁基态和磁场历史调控的 大交换偏置效应}

廖晓奇 ${ }^{1,2}$, 高禄梅 ${ }^{3}$, 王宇 ${ }^{1 *}$, 徐至金, Muhammad Tahir Khan ${ }^{4}$, 常铁严 ${ }^{1}$, 陈凯运 ${ }^{1}$, 曾昱嘉 ${ }^{2}$, 杨森 ${ }^{1 *}$, Peter Svedlindh ${ }^{5}$

摘要 由于交换偏置效应对磁记录和自旋电子器件的重要意义, 在磁 性形状记忆合金中获得大偏置场一直是人们长期努力的目标. 本文 通过调控 $\mathrm{Ni}_{50} \mathrm{Mn}_{34} \mathrm{In}_{16-x} \mathrm{Fe}_{x}(x=1,3,5$ )磁性形状记忆合金的磁性基 态(取决于合金成分)和磁场历史 (取决于冷却过程中冷却场 $H_{\mathrm{FC}}$ 和等 温磁化过程中所施加的最大测试场 $H_{\mathrm{Max}}$ ), 对交换偏置行为进行了系 统研究. 结果表明, 调控磁性基态和磁场历史可以获得最大铁磁簇与 反铁磁基体界面体积分数以及它们之间的强相互作用, 从而诱发了 界面上强单向各向异性, 产生了大交换偏置场. 本文提出了获得大交 换偏置场需要遵循的两条原则. 首先, 选择稀磁自旋玻璃与强反铁磁 基体的磁性基态成分可获得大交换偏置场; 其次, 通过增强 $H_{\mathrm{FC}}$ 和降 低 $H_{\mathrm{Max}}$ 有利于实现大交换偏置. 本研究为设计具有大交换偏置场的 非均匀磁性相化合物提供了一种有效的方法. 